\title{
Comparison Between Standard and Gender-Specific Knee Designs in Total Knee Arthroplasty in Female Patients
}

\author{
Elsayed Morsey Zaki ${ }^{1}$, Tarek Ali Elkhadrawe ${ }^{2}$, Ayman Mohammed Ebied ${ }^{1}$, Amro Saber Elsayed ${ }^{1}$, \\ Hany Elsayed Saad, *
}

${ }^{1}$ Department of Orthopedic surgery, Faculty of medicine, Menoufia University, Menoufia, Egypt

${ }^{2}$ Department of Orthopedic surgery, Faculty of medicine, Alexandria University, Alexandria, Egypt

\section{Email address:}

hanysaad30@yahoo.com (H. E. Saad)

\section{To cite this article:}

Elsayed Morsey Zaki, Tarek Ali Elkhadrawe, Ayman Mohammed Ebied, Amro Saber Elsayed, Hany Elsayed Saad. Comparison Between Standard and Gender-Specific Knee Designs in Total Knee Arthroplasty in Female Patients. American Journal of Sports Science.

Vol. 3, No. 4, 2015, pp. 67-72. doi: 10.11648/j.ajss.20150304.11

\begin{abstract}
Objectives: Current study is a comparative, prospective, randomized, short-term outcome study to compare the early results of Total Knee Arthroplasty in female patients using either standard or gender-specific knee prosthesis for treatment of advanced osteoarthritis of the knee joint. Background: Gender-specific knee athroplasty prosthesis is designed to better accommodate the differences noted in distal femoral anatomy in female patients compared to males. Several studies have reported differences in knee morphometry between genders such as the height/width ratio of the distal femoral condyle, the quadriceps angle and the shape of the distal femur. The need for Gender-specific (GS) femoral prostheses is still debated and has led us to compare short-term outcomes of TKA using standard (STD) or GS femoral components. Methods: In the period between February 2012 and February 2013, a comparative prospective randomized study was conducted involving 34 female patients with 40 knees who underwent primary total knee arthroplasty for knee joint advanced osteoarthritis and divided into two groups, The first group included 20 knees underwent total knee arthroplasty using Gender-Specific Knee Design with Gender-Specific femoral component (Zimmer Gender Solutions NexGen High-Flex ( NexGen LPS-Flex ) Implant, The second group included 20 knees underwent total knee arthroplasty using standard Knee Design with Standard femoral component (Zimmer NexGen LPS Implant). Results: No statistically significant differences were observed between the two groups regarding pain improvement, range of motion improvement, pre and post operative OXFORD scores, Knee Society Scores, WOMAC scores, satisfaction, preference, complications, and radiographic results. Conclusion: Early clinical outcomes for the knees with a gender-specific NexGen LPS-Flex prosthesis were similar to those for the knees with a standard NexGen LPS prosthesis in female patients. gender-specific knee prosthesis showed no advantages over standard unisex knee prosthesis in terms of early clinical outcomes.
\end{abstract}

Keywords: Gender, Gender-Specific, Arthroplasty, Knee

\section{Introduction}

Total Knee Arthroplasty (TKA) is a successful procedure with long-term durability. Overall, survivorship is $91 \%$ at 10 years, $84 \%$ at 15 years, and $78 \%$ at 20 years. TKA is considered one of the most successful health care interventions for end-stage arthritis of the knee. Successful total knee arthroplasty results in pain relief, knee function improvement and improved quality of life. ${ }^{1,2}$

The number of patients undergoing TKA surgery has been continuously increasing and has displayed a higher proportion of female patients. In a study of more than 48,000
Canadians aged 55 years and older, the number of individuals with the potential need for hip or knee arthroplasty was estimated to be 44.9 per 1,000 women and 20.8 per 1,000 men. ${ }^{3}$ Women typically represent approximately $60 \%$ of patients undergoing TKA. ${ }^{4,5,6}$

Women dominate both the population that needs TKA and the currently treated patient population. Thus, there is a strong rationale for evaluating female-specific knee requirements based on sex differences in joint anatomy and kinematics. $^{7}$

Differences in anatomy and morphometry of knee between men and women have been well identified. Several studies 
have reported differences in knee morphometry between genders such as the height/width ratio of the distal femoral condyle, the quadriceps angle ( $\mathrm{Q}$ angle) and the shape of the distal femur. ${ }^{8,9,10}$

Morphological data indicate that women tend to have narrower medial to lateral dimension of femoral condyle for any given anterior to posterior dimension. $Q$ angle is significantly greater in female population than in male population. The anterior femoral condylar anatomy is more pronounced in male knees.

The recent introduction gender-specific knee arthroplasty implants was a new approach to the ongoing trend across TKA systems to offer more sizing options and is based on the anatomic differences between male and female femurs. Rather than simply offering more sizes with similar AP to ML ratios, the gender-specific component is designed to better accommodate the anatomic differences noted in females with a narrower ML dimension for any given AP dimension. In addition, the angle of the trochlear groove was increased and the anterior flange thickness was reduced to better match the native female anatomy. $7,11,12$

This gender-specific implant has theoretical advantage over standard TKA implants, whether female patients can benefit from this system is under debate. Thus, we conducted this study to evaluate the early clinical outcomes between two implants.

\section{Material and Method}

In the period between February 2012 and February 2013, a comparative prospective randomized study was conducted involving 34 female patients with 40 knees who underwent primary total knee arthroplasty for knee joint advanced osteoarthritis and divided into two groups, The first group included 20 knees underwent total knee arthroplasty using Gender-Specific Knee Design with Gender-Specific femoral component (Zimmer Gender Solutions NexGen High-Flex (NexGen LPS-Flex) Implant, The second group included 20 knees underwent total knee arthroplasty using standard Knee Design with Standard femoral component (Zimmer NexGen LPS Implant).

\section{Inclusion and Exclusion Criteria}

All patients with primary degenerative or inflammatory varus osteoarthritis with tibio - femoral angle more than 0 degrees, aged above 50 years and their deformities are totally articular i.e. wear and erosion of the medial compartment of the joint were included. Male patients, female patients with valgus osteoarthritis, post traumatic osteoarthritis, patients with a history of open knee surgery that required metallic implant placement, patients with a bone defect that required bone grafting or metallic augmentation, and revision cases were excluded

The mean age of two groups of patients included at the time of surgery was 63 years old (range from 50 to 72 years), 21 right knee replaced, 19 left knee replaced, while 6 patients had bilateral total knee replacement 1 of them were done bilateral simultaneous at the same setting.

Medial parapatellar approach was used in all cases. Bone cuts were performed in a standard manner with the distal femoral and proximal tibial bone cuts perpendicular to the mechanical axis in the coronal plane. The proximal tibial cut incorporated a posterior slope of approximately 7 in the sagittal plane.

Femoral component sizing was performed with a posterior referencing instrument that sets the resection of the posterior condyles equal to the thickness of the component to attempt to accurately restore the posterior condylar offset. This guide also incorporates an anterior boom that allows secondary referencing of the anterior cortex that helps prevent notching. The femoral component rotation was set parallel to the transepicondylar axis.

After cutting distal femoral cut, we re-checked femoral sizing to choose the correct size of femoral prosthesis that avoids mediolateral verhanging. In cases with standard prosthesis , After measuring anteroposterior size of the distal femur, we checked mediolateral size of the trial prosthesis, and if it was obvious overhanging medially we moved to the smaller size femoral component ( downsizing), so we had no patient with mediolateral overhang and accepted mediolateral underhang in a in about $20 \%$ of standard group prosthesis. In cases with gender specific prosthesis we observed no overhanging of any case when using trial component of the correct size, but observed undercoverage in upto $70 \%$ of gender specific group.

If downsizing was required based on intraoperative assessment that the desired femoral component was too wide, additional posterior resection was performed. In most cases, some compromise was required and included the surgeon's assessment of the extent of the overhang, flexion and extension gap balance, and remaining anterior bone that could be resected before producing a notch.

\section{Results}

In the current study, we found that the early clinical outcomes for the knees with a gender-specific NexGen LPSFlex prosthesis were similar to those for the knees with a standard NexGen LPS prosthesis. In both groups, female patients had improved quality of life in terms of pain, range of motion of the knee, walking distance, deformity correction , OXFORD , KSS ,KSS-F , WOMAC scores, and function after total knee arthroplasty. We also found negligible differences in terms of patient satisfaction and preference between the two prostheses in five bilateral cases.

\subsection{Range of Motion (ROM)}

In current study mean preoperative ranges of knee motion in the supine position were 110 and 108 degrees in the gender-specific and standard implant groups, respectively. The mean postoperative ranges of knee motion in the supine position were 115 and 113 degrees, respectively. mean improvement in flexion range of both groups was 5 degrees 
(range from 0 to 25 ) with no statistically significant difference between two groups.

\subsection{Knee Society Score (KSS)}

In current study mean preoperative KSS were 27 and 25 points in the gender-specific and standard implant groups, respectively. The mean postoperative KSS were 83 and 84 points, respectively. mean improvement in KSS were 55 and 59 points, respectively with no statistically significant difference between two groups.

Pain improvement in most patients from severe ( score 0 in KSS ) to mild occasional pain( score 45 in KSS ) or no pain ( score 50 in KSS ) was the leading cause of such improvement in score. Other items improvement such as range of motion, mediolateral stability and deformity correction played minor role in score improvement as each improvement in range of motion by 5 degrees improve the score only 1 point and most of patients included in this study had mild to moderate varus deformity with mild to moderate lateral side laxity.

\subsection{Functional Knee Society Score (KSS-F)}

In current study mean preoperative KSS-F were 28 and 26 points in the gender-specific and standard implant groups, respectively. The mean postoperative KSS-F were 83 and 82 points, respectively. mean improvement in KSS-F were 54 and 56 points, respectively with no statistically significant difference between two groups.

\subsection{WOMAC Score}

In current study mean preoperative WOMAC score was 81 points for both groups while mean postoperative WOMAC score was 27 and 29 points in the gender-specific and standard implant groups, respectively. with no statistically significant difference between two groups.

\subsection{Prosthesis Fitness}

We had no patient with mediolateral overhang. After measuring anteroposterior size of the distal femur, we checked mediolateral size of the trial prosthesis, and if it was obvious overhanging medially we moved to the smaller size femoral component, so we had no patient with mediolateral overhang and accepted mediolateral underhang in a in about $20 \%$ of standard group prosthesis.

We observed no overhanging of any case with gender specific prosthesis when using trial of the correct size but observed undercoverage in upto $70 \%$ of gender specific group (fig 1,2 )

\subsection{Radiographic Findings}

Femoral component undercoverage of distal femur in mediolateral dimensions was observed in anteroposterior view of some cases of first group. (fig -3)

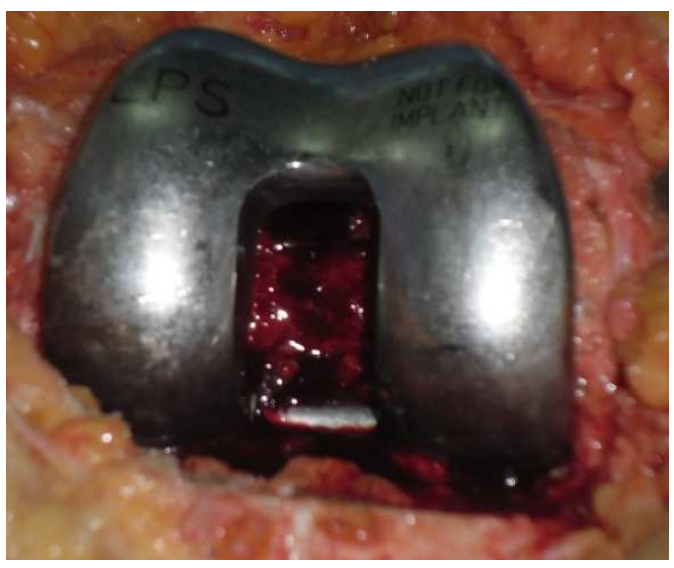

Fig. 1. Stadnadr prosthesis trial.

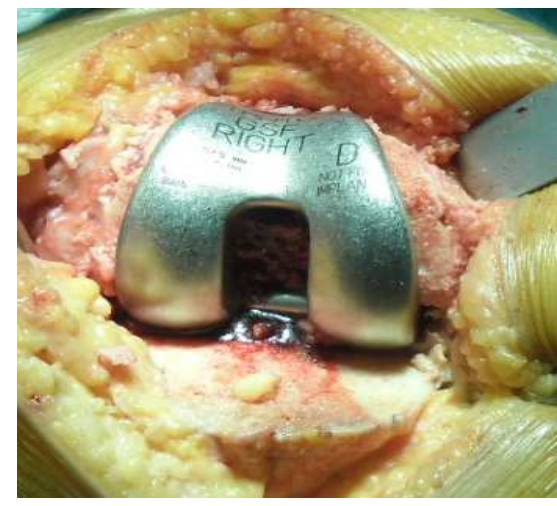

Fig. 2. Gender-specific prosthesis trial showing under coverage of medial side.

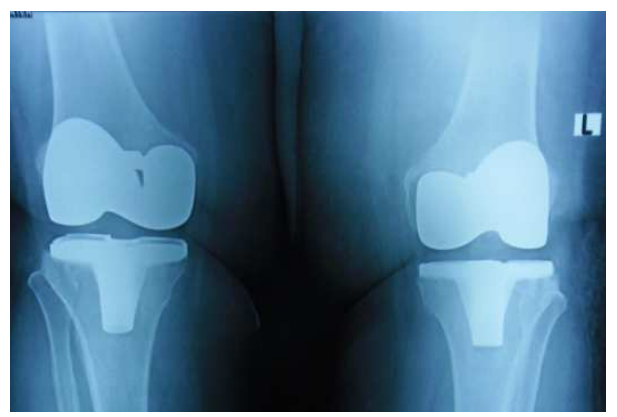

Figure 3. The femoral component of the gender-specific prosthesis appears to be undersized( left side).

\section{Discussion}

A gender-specific total knee prosthesis has been introduced to match the three notable anatomic differences in the female population: a less prominent anterior condyle, an increased quadriceps angle ( $\mathrm{Q}$ angle), and a reduced mediolateral: anteroposterior aspect ratio. 7,12

A NexGen gender-specific femoral component (Zimmer gender-specific NexGen LPS-Flex) was designed with a narrow mediolateral dimension for a given anteroposterior dimension to more closely match the aspect ratio in the knees of female patients. The anterior flange of the gender-specific femoral component was modified to include a recessed patellar sulcus and reduced anterior condylar height (to 
account for a less pronounced anterior condyle in women) and a lateralized patellar sulcus (to accommodate the increased $Q$ angle associated with a wider pelvis).,12

The need for gender-specific knee arthroplasties is based on the following assumptions:

(1) women have results that are inferior to those of men after TKA, and

(2) traditional prosthetic designs have failed to address anatomical differences between genders .

A number of clinical studies have refuted the idea that women have worse outcomes than men using traditional total knee designs. ${ }^{13,14,15,16}$ In fact, some studies have found that women achieve essentially the same results as men, or even better. ${ }^{14,17,18}$

A systematic review by Merchant et al. found no evidence for anatomical differences between men's and women's knees that would justify a female-specific design. The average anatomical differences between male and female knees can be explained by the smaller height and size of women on average, not by their gender ${ }^{14}$

A recent study showed that the shape of the knee is not only dependent on gender, but also on the morphotype of the patient. ${ }^{19}$

However, these anatomical differences may be so small that have no clinical effect, which would agree with the findings of the present study.

Cheng et al conducted systematic review and metaanalysis of the current literature up to January 2013, they performed an electronic and manual search of of all available current world literature related to results of TKA to evaluate difference between gender specific and standard unisex TKA

Only 6 RCTs met the author,s eligibility criteria ( Kim et al. 2010 a , b , Kumar et al. 2012, Singh et al. 2012, Song et al. 2012 , Thomsen et al. 2012 ). ${ }^{21,22,23,24,25,26}$

They included 846 knees, in which 423 female patients had bilateral TKA with a gender-specific prosthesis in one knee and a standard unisex prosthesis in the contralateral knee.

Of the 6 studies, 3 were conducted in Korea (Kim et al. 2010 a , b, Song et al. 2012 ), 2 in India (Kumar et al. 2012, Singh et al. 2012), and one in Denmark (Thomsen et al. 2012).

All included studies used Zimmer NexGen Implants (Zimmer Inc, Warsaw, Indiana) for all knees . Of the 6 studies, 2 used NexGen cruciate retaining prosthesis i.e. GS CR-Flex in one side and CR -Flex in the other side (Kim et al. 2010 b, Song et al. 2012) and 4 used NexGen cruciate substituting prosthesis i.e. GS LPS-Flex in one side and NexGen LPS in the other side (Kim et al. 2010a, Singh et al. 2012, Thomsen et al. 2012 Kumar et al. 2012 ).

Results of 6 mentioned studies are in general similar to results of current study as they found no statistically significant differences were observed between the 2 designs regarding pain improvement, range of motion, knee scores, satisfaction, preference, complications, and radiographic results.

The gender-specific design reduced the prevalence of overhang. However, it had less overall coverage of the femoral condyles compared to the unisex group. In fact, the femoral prosthesis in the standard unisex group matched better than that in the gender-specific group.

One of the main design features of gender-specific knee is reduced anterior flange thickness to decrease prevalence of Overstuffing of the patellofemoral compartment.

Overstuffing of the patellofemoral compartment may be associated with pain and reduced ROM. The use of a standard TKA could possibly lead to overstuffing because of a less prominent anterior condyle in the female knee.

Despite the fact that reduced height of the anterior flange and the deeper trochlear groove would help to prevent overstuffing of the patellofemoral joint and improve patellar tracking in the gender- specific design, current study showed similar postoperative pain and ROM when comparing the two knee prostheses.

Another design feature of the gender-specific prosthesis is the trochlear groove angle of the femoral component, which is increased by approximately 3 degrees in order to replicate the distinct $\mathrm{Q}$ angle difference, thereby enhancing patellar tracking and reducing the need for lateral retinacular release. In the current study, the patellar tilt angle did not differ significantly between the two groups either preoperatively or postoperatively. No knee in either group had subluxation or dislocation of the patella or underwent a retinacular release.

One of the main design features of gender-specific knee is modified mediolateral dimensions to better accommodate distal femoral morphology of female femora.

Meta-analysis of 3 trials revealed that the gender-specific femoral component did not fit better than the standard femoral component (Kim et al. 2010a,b, Kumar et al. 2012, Thomsen et al. 2012) .

In the female patients with the standard prosthesis, the femoral prosthesis was closely matched in 172 knees (56\%), overhung in $41(13 \%)$, and undercovered the bone in 94 $(31 \%)$. In the female patients with the gender-specific prosthesis, 77 knees $(25 \%)$ had a close fit and 230 knees (75\%) had undercoverage.

Kim et al. found that the gender-specific NexGen LPSFlex femoral component did not fit better than the standard NexGen LPS femoral component did. There was a significant association between the component type and the amount of overhang or underhang in the standard or genderspecific prosthesis $(\mathrm{p}<0.0001)$.

In the group with a standard implant, the mediolateral and anteroposterior measurements closely approximated the distal femoral morphologic data for fifty-one knees (60\%). Ten knees (12\%) had an overhang (1 to $3 \mathrm{~mm}$ ), and twentyfour knees (28\%) had an underhang (range, 1 to $2 \mathrm{~mm}$ ).

In the group with a gender-specific implant, fourteen knees (16\%) had a close fit and seventy-one knees (84\%) had an underhang (mean, $2.8 \pm 1.3 \mathrm{~mm}$; range, 1 to $7 \mathrm{~mm}$ ).

Kumar et al. reported that in those with a standard prosthesis, the femoral component was closely matched in 30 knees, overhung in 17 and undercovered the bone in 13. In those with a gender-specific prosthesis, it was closely 
matched in 45 knees and undercovered the bone in 15 .

In the current study there was similar observation, but we had no patient with mediolateral overhang. After measuring anteroposterior size of the distal femur, we checked mediolateral size of the trial prosthesis, and if it was obvious overhanging medially we moved to the smaller size femoral component, so we had no patient with mediolateral overhang and accepted mediolateral underhang in a in about $20 \%$ of standard group prosthesis.

In the current study, it was observed that no overhanging of any case with gender specific prosthesis when using trial of the correct size but observed undercoverage in upto $70 \%$ of gender specific group. This means that we observed the standard prosthesis fitted the distal femur in Egyptian females better than gender specific prosthesis in terms of mediolateral dimensions, but gender specific prosthesis fitted the distal femur better in terms of antero posterior dimensions, thinner anterior flange and better patella femoral tracking and reduction of patellofemoral overstuffing. (fig $1,2,3)$

Tanavalee et al. ${ }^{27}$ intraoperatively evaluated the overhang of the cutting block on the posteromedial edge of the femoral condyle. They found that the overall percentage of genderspecific prosthesis selection was higher in female patients than in male patients. This phenomenon frequently occurs in medium-sized to large-sized female patients. The medial or lateral overhang of unisex prostheses was worse in female knees than in male knees. ${ }^{28}$ Yan et al. reported a higher prevalence of prosthetic overhang in women with standard unisex knee prostheses ${ }^{29}$

Consistent with these findings, current study showed that gender-specific design reduced medial or lateral overhang in female patients whereas there was a higher prevalence of underhang than with unisex prostheses. current study result is consistent with results of Kim et al. 2010a,b, Kumar et al. 2012, Thomsen et al. 2012 regarding fit of femoral component to distal femur .

Although a Mahoney and Kinsey ${ }^{30}$ study confirmed that femoral component overhang may create postoperative pain due to soft tissue irritation and soft tissue imbalance, results of previously mentioned studies suggest that the difference in condylar coverage does not affect pain scores and knee function.

Based on these contradictory results, the question remains whether the modifications made to the gender-specific design are extensive enough to closely match femoral anatomy in female patients and offer any clinical advantages.

Theoretically, the higher incidence of underhang in the gender-specific group, which exposed more cancellous bone than with the NexGen standard prostheses, could be a source of higher perioperative blood loss and may induce increased ostolysis from wear debris with longer follow-up. ${ }^{8,21,22}$

\section{Conclusion and Recommendations}

It was observed that with standard prosthesis in about $20 \%$ of female cases, surgeon either accept mediolateral overhanging with its potential soft tissue irritation and post operative pain or downsize femoral component with its potential over resection of posterior femoral condyles , flexion-extention mismatch and mid flexion instability .

With gender-specific knee, in most cases (about $70 \%$ ) surgeon must accept mediolateral undercoverage (varying from 2 to 10 millimeters) when using correct anteroposterior size. Gender-specific prosthesis better restores the quadriceps (Q) angle and better restores size of anterior femoral condyles of female patients which is more prominent in males than females.

To achieve best antero-posterior size and best mediolateral coverage surgeon needs the two sets of standard and genderspecific prosthesis available when performing TKA for a female patient.

Its recommended either to provide gender specific prosthesis with wider medio-lateral dimension for middle east and African females or provide standard prosthesis with variable aspect ratios for each antero-posterior size to overcome the problems of medio-lateral overhanging or undercoverage which is expected to achieve better matching in all cases.

Problems of previous two solutions are the large number of instruments and large number of implants needed to be available to perform TKA.

There may be a future solution for these mismatching problems by future development of patient specific instruments and patient specific implants depending on accurate analysis of preoperative computerized images.

Almost all prosthetic implants have been designed and manufactured to accommodate the knee anatomy of Western Caucasians. Anthropometric studies for distal femoral anatomy of Egyptian patients are needed to establish refrences for normal aspect ratio, distal femoral rotation and proximal tibial sizes, slope and rotational alignment which proved to show racial, ethnic, gender and individual differences.

\section{References}

[1] Rand JA, Trousdale RT, Ilstrup DM, Harmsen WS: Factors affecting the durability of primary total knee prostheses. J Bone Joint Surg Am 2003;85-A:259-265

[2] Bourne RB, Chesworth BM, Davis AM, Mahomed NN, Charron KD (2010) Patient satisfaction after total knee arthroplasty: who is satisfied and who is not? Clin Orthop Relat Res 468(1):57-63

[3] Hawker GA, Wright JG, Coyte PC, et al: Differences between men and women in the rate of use of hip and knee arthroplasty. N Engl J Med 2000; 342:1016-1022.

[4] Rand JA, Ilstrup DM .Survivorship analysis of total knee arthroplasty: cumulative rates of survival of 9200 total knee arthroplasties. J Bone Joint Surg [Am] (1991). 73-A:397-409

[5] Ritter MA, Wing JT, Berend ME, Davis KE, Meding JB (2008) The clinical effect of gender on outcome of total knee arthroplasty. J Arthroplast 23:331-336 
[6] Font-Rodriguez DE, Scuderi GR, Insall JN. Survivorship of cemented total knee arthroplasty. Clin Orthop 1997; 345:79.

[7] Conley S, Rosenberg A, Crowninshield R. The female knee: anatomic variations. J Am Acad Orthop Surg. 2007; 15(suppl1):S31-S36.

[8] Hitt K, Shurman JR II, Greene K, et al: Anthropometric measurements of the human knee: Correlation to the sizing of current knee arthroplasty systems. J Bone Joint Surg Am 2003; 85(suppl 4):115-122.

[9] Lonner JH, Jasko JG, Thomas BS. Anthropomorphic differences between the distal femora of men and women. Clin Orthop Relat Res. 2008; 466:2724-2729.

[10] Fehring TK, Odum SM, Hughes J, Springer BD, Beaver Jr WB. Differences between the sexes in the anatomy of the anterior condyle of the knee. J Bone Joint Surg Am 2009; 91:2335-41.

[11] Booth RE Jr. Sex and the total knee: gender-sensitive designs. Orthopedics. 2006; 29:836-838.

[12] Greene Kenneth A. Gender-Specific Design in Total Knee Arthroplasty 2007 The Journal of Arthroplasty Vol. 22 No. $7: 27-31$

[13] MacDonald SJ, Charron KD, Bourne RB, Naudie DD, McCalden RW,Rorabeck CH: The John Insall Award: genderspecific total knee replacement: prospectively collected clinical outcomes. Clin Orthop RelatRes 2008, 466:2612-2616

[14] .Merchant A C, Arendt E A, Dye S F, Fredericson M, Grelsamer R P, Leadbetter W B, Post W R, Teitge R A. The female knee: anatomic variations and the female-specific total knee design. Clin Orthop 2008; (466) (12): 3059-65.

[15] Dalury D F, Mason J B, Murphy J A, Adams M J. Analysis of the outcome in male and female patients using a unisex total knee replacement system. J Bone Joint Surg (Br) 2009; 91 (3): 357-60.

[16] Johnson A J, Costa C R, Mont M A. Do we need genderspecific total joint arthroplasty? Clin Orthop 2011; (469) (7): 1852-8.

[17] O'Connor M I. Implant survival, knee function, and pain relief after TKA: are there differences between men and women?" Clin Orthop 2011; (469) (7): 1846-51.

[18] Parsley B S, Bertolusso R, Harrington M, Brekke A, Noble P C. Influence of gender on age of treatment with TKA and functional outcome. Clin Orthop 2010; (468) (7): 1759-64.

[19] Bellemans J, Carpentier K, Vandenneucker H, et al. The John
Insall Award: both morphotype and gender influence the shape of the knee in patients undergoing TKA. Clin Orthop Relat Res. 2010; 468:29-36.

[20] Cheng CK, Liau JJ. Morphometrical measurements of resected surface of femurs in Chinese knees: correlation to the sizing of current femoral implants. Knee 2006; 13:12.

[21] Kim Y H, Choi Y, Kim J S. Comparison of a standard and a gender-specific posterior cruciate-substituting high-flexion knee prosthesis: a prospective, randomized, short-term outcome study. J Bone Joint Surg (Am) 2010 a; 92 (10): 191120.

[22] Kim Y H, Choi Y, Kim J S. Comparison of standard and gender-specific posterior-cruciate-retaining high-flexion total knee replacements: a prospective,randomised study. J Bone Joint Surg (Br) 2010 b; 92 (5): 639-645.

[23] Kumar V, Bhavuk G, Malhotra R. A randomized trial comparing gender specific with gender non-specific knee arthroplasty implants.J Bone Joint Surg (Br) 2012; 94: 525.

[24] Singh H, Mittal V, Nadkarni B, Agarwal S, Gulati D. Genderspecific highflexion knee prosthesis in Indian women: a prospective randomised study.J Orthop Surg (Hong Kong) 2012; 20 (2): 153-6.

[25] Song E K, Jung W B, Yoon T R, Park K S, Seo H Y, Seon J K. Comparison of outcomes after bilateral simultaneous total knee arthroplasty using genderspecific and unisex knees. J Arthroplasty 2012; 27 (2): 226-31.

[26] Thomsen M G, Husted H, Bencke J, Curtis D, Holm G, Troelsen A. Do we need a gender-specific total knee replacement? A randomised controlled trial comparing a highflex and a gender-specific posterior design. J Bone Joint Surg (Br) 2012; 94 (6): 787-92.

[27] Tanavalee A, Rojpornpradit T, Khumrak S, Ngarmukos S (2011) The early results of gender-specific total knee arthroplasty in Thai patients. Knee 18(6):483-487

[28] Clarke H D, Hentz J G. Restoration of femoral anatomy in TKA with unisex and gender-specific components. Clin Orthop 2008; (466) (11): 2711-6.

[29] Yan M, Wang J, Wang Y, Zhang J, Yue B, Zeng Y. Genderbased differences in the dimensions of the femoral trochlea and condyles in the Chinese population: Correlation to the risk of femoral component overhang. Knee 2014; 21(1):2 52-6.

[30] Mahoney O M, Kinsey T. Overhang of the femoral component in total knee arthroplasty: risk factors and clinical consequences. J Bone Joint Surg (Am) 2010; 92 (5): 115-21. 